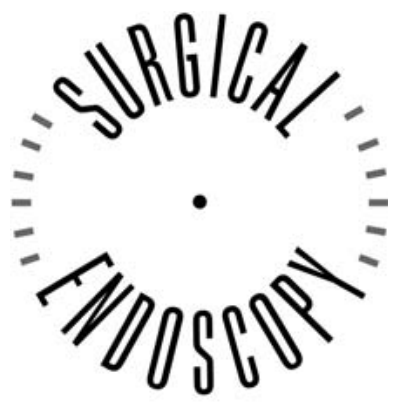

and Other Interventional Techniques

\title{
The influence of different training schedules on the learning of psychomotor skills for endoscopic surgery
}

\author{
E. G. G. Verdaasdonk, ${ }^{1,2}$ L. P. S. Stassen, ${ }^{1,2}$ R. P. J. van Wijk, ${ }^{1}$ J. Dankelman ${ }^{1}$ \\ ${ }^{1}$ Department of BioMechanical Engineering, Faculty of Mechanical, Maritime, and Materials Engineering, Man-Machine Systems Group, Delft \\ University of Technology, Mekelweg 2, 2628 CD Delft, The Netherlands \\ ${ }^{2}$ Department of Surgery, Reinier de Graaf Group, Delft, The Netherlands
}

Received: 19 December 2005/Accepted: 24 May 2006/Online publication: 21 November 2006

\begin{abstract}
Background: Psychomotor skills for endoscopic surgery can be trained with virtual reality simulators. Distributed training is more effective than massed training, but it is unclear whether distributed training over several days is more effective than distributed training within 1 day. This study aimed to determine which of these two options is the most effective for training endoscopic psychomotor skills.

Methods: Students with no endoscopic experience were randomly assigned either to distributed training on 3 consecutive days (group A, $n=10$ ) or distributed training within 1 day (group $\mathrm{B}, n=10$ ). For this study the SIMENDO virtual reality simulator for endoscopic skills was used. The training involved 12 repetitions of three different exercises (drop balls, needle manipulation, $30^{\circ}$ endoscope) in differently distributed training schedules. All the participants performed a posttraining test (posttest) for the trained tasks 7 days after the training. The parameters measured were time, nontarget environment collisions, and instrument path length.

Results: There were no significant differences between the groups in the first training session for all the parameters. In the posttest, group A (training over several days) performed $18.7 \%$ faster than group B (training on 1 day) ( $p=0.013)$. The collision and path length scores for group A did not differ significantly from the scores for group B.

Conclusion: The distributed group trained over several days was faster, with the same number of errors and the same instrument path length used. Psychomotor skill training for endoscopic surgery distributed over several days is superior to training on 1 day.
\end{abstract}

Key words: Endoscopic — Laparoscopic — Psychomotor skills - Training — Training schedules - VR simulator

Correspondence to: E. G. G. Verdaasdonk
In recent years, training of psychomotor skills for endoscopic surgery has been shifted from the operation theater to the skills laboratory. To overcome the difficulties in performing endoscopy such as disturbed hand-eye coordination, visual feedback from a threedimensional (3D) environment to a 2D monitor, and working with long instruments, the surgeon has to practice. Several studies have shown that virtual reality (VR) simulators are useful and valid tools for training psychomotor skills such as hand-eye coordination $[5,6$, $8,9,13,14]$. Currently, the training of these skills generally is undertaken in structured courses during 1 or 2 days and continues inside the clinic. With the reduced trainee working hours and the increased pressure on the use of health care facilities, training time needs to be used efficiently. It is therefore important to know how long students should train, when they should train, and what influence different training schedules has on the performance.

The effect of different training schedules with respect to their distribution has been studied in other fields such as psychology and neuroscience $[1,4,11]$. Distributed training refers to a practice schedule in which periods of training are interspersed with rest periods. Massed practice refers to a continuous block of training. Meta-analytic reviews indicate that distributed training results in a better retention of motor skills than massed training. However, the authors of the reviews also state that the magnitude of the distributed practice effect depends highly on the tasks trained [11]. Furthermore, the skills studied in the mentioned reviews involved simple motor behavior, and not the less intuitive skills involved in endoscopic surgery (e.g., disturbed eye-hand coordination). Only one study, by Mackay et al. [12], found that distributed endoscopic motor skills training with short breaks (several minutes) is superior to massed training (no breaks) within one single day. However, it is not clear whether training distributed over several days is more effective than 


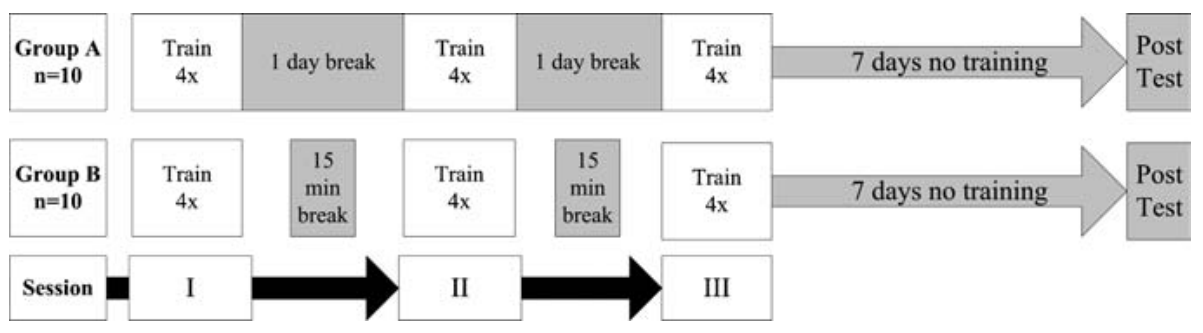

Fig. 1. Training schedule for groups A (distributed training over several days) and $\mathrm{B}$ (distributed training on 1 day). training distributed over one single day. Furthermore, it is unclear whether the effect can already be measured at the end of the training, or whether it is measurable only after several days.

Evidence from a neurologic study showed that one night of sleep after motor skills training enhanced the activity of motor areas in the brain [18]. This suggests that sleep can enhance the learning effect for motor skills needed to perform endoscopic surgery.

The goal of this study was to determine the most effective schedule for training psychomotor skills needed to perform endoscopic surgery using a VR simulator: distributed training over several days or distributed training on 1 day.

\section{Methods}

Students who had no prior experience with endoscopic surgery or endoscopic skills training were recruited from the Faculty of Medicine and Health Sciences of the Erasmus University of Rotterdam and the Delft University of Technology. These students were randomly assigned to two groups of 10 subjects each: group A (training over several days) and group B (training on 1 day with short breaks).

All the participants filled out a questionnaire about their personal characteristics (age, gender, dominant hand, and educational background), prior experience (VR games and musical instruments), motivation to participate (rated on a scale of 1 to 10), and dexterity (self-rated on a scale of 1 to 10) in terms of how they would perform (performance prediction) on the simulator. In the rating scales used, 1 was considered the lowest and 10 the highest score for own motivation, dexterity, and performance prediction.

For this study, the SIMENDO VR simulator (Delltatech, Delft, The Netherlands) was used. Previously, the face and content validity of this simulator was established for the single-instrument exercises, and the first results for construct validity were shown [16]. The SIMENDO is a low-fidelity VR simulator developed to train basic endoscopic skills, such as eye-hand coordination, with the use of abstract tasks. The hardware of the simulator in this study consisted of two separate instruments connected to a laptop with a USB cable. The 15.4-in. screen was placed on a table in front of the participant behind the hardware of the simulator. The training took place in a quiet room and was observed by one or two researchers.

The participants were trained with the following tasks: drop the balls (picking and placing three balls in holes using one instrument in the right hand), the ring (passing a needle through two rings using two instruments, one in each hand), and $30^{\circ}$ endoscope handling (picking and placing four balls on a box with an instrument in the right hand and a camera in the left hand). The parameters measured were time to completion of the task (seconds), collisions of instruments with the nontarget environment $(n)$, and the path length of the right and left instrument (arbitrary units).

The training for group A consisted of 12 repetitions divided over three consecutive days. Thus, each day, a training session of four repetitions was performed. Group B performed 12 repetitions within a single day. After each session of four repetitions, the group B participants had a 15-min break. Figure 1 displays the training schedules for both groups. Each repetition was measured and automatically
Table 1. Characteristics of the group A (training on several days) and group B (training on one day) participants

\begin{tabular}{lll}
\hline Characteristics & Group A & Group B \\
\hline $\begin{array}{l}\text { General } \\
\text { Median age in years: } n \text { (range) }\end{array}$ & $21(19-27)$ & 21 (19-25) \\
$\begin{array}{l}\text { Male:female } \\
\text { Left-hand dominance }(n)\end{array}$ & $6: 4$ & $6: 4$ \\
$\begin{array}{l}\text { Medical background }(n) \\
\text { Prior experience }\end{array}$ & 8 & 2 \\
$\begin{array}{l}\text { Some experience with VR games (n) } \\
\text { Playing a musical instrument }(n)\end{array}$ & 4 & 8 \\
$\begin{array}{l}\text { Motivation to learn skills for } \\
\quad \text { endoscopic surgery (self-rating) }\end{array}$ & 6 & 7 \\
$\begin{array}{l}\text { Median rate: } n \text { (range) } \\
\text { Dexterity (self-rating) }\end{array}$ & $8(8-10)$ & 5 \\
$\begin{array}{l}\text { Median rate: } n \text { (range) } \\
\text { Performance prediction (self-rating) }\end{array}$ & $7(7-10)$ & $6.5(5-9)$ \\
Median rate: $n$ (range) & $6(6-7)$ & $6(6-7)$ \\
\hline
\end{tabular}

VR, virtual reality

${ }^{\text {a }}$ Rating on a scale of 1 (lowest score) to 10 (highest score)

stored in a Microsoft Access 2002 database connected to the simulator software.

A posttraining test (posttest) was performed 7 days after the training. For this test, the same tasks performed in the training were used and measured for one repetition. The total number of repetitions $(n=12)$ and the interval between the end of the last training session and the posttest ( 7 days) were equal for the two groups.

\section{Statistical analysis}

The answers on the questionnaires were compared between the two groups. The parameter scores for all the tasks were summed for each repetition and analyzed. Subsequently, the parameter scores also were analyzed for each task separately per repetition and compared between the two groups. Differences between groups were tested for statistical significance using the Mann-Whitney $U$ test for nonparametric data.

\section{Results}

Table 1 shows the results for the answers to the questionnaire. There were no differences between the characteristics and the ratings of the two groups.

Figure 2 shows the results in box plots of the total time score (s) for all three tasks summed per repetition for both groups. Figure 3 shows the results at the end of the training (end training) and the posttest results with respect to time (s).

Table 2 presents the total scores for the first training cycle, the end of the training, and the posttest. In the posttest, group A (training over several days) performed $18.7 \%$ faster than group B (training on 1 day) 


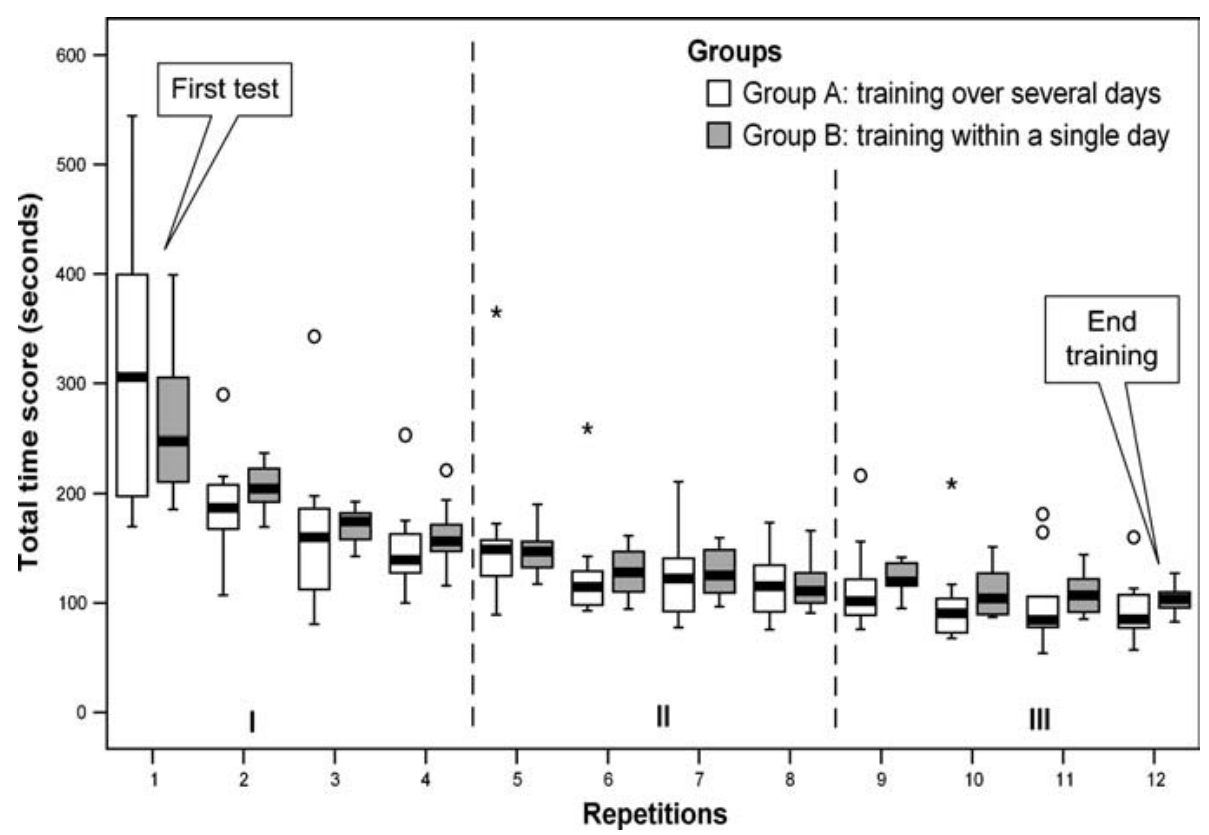

Fig. 2. Box plots of time scores summed for all three tasks showing the difference between groups A (training over several days) and $\mathrm{B}$ (training within a single day). The boxes represent the interquartile range. The bars medians and whiskers represent the first percentile range excluding outliers. The circles represent outliers, and the asterisks represent extreme outliers. Roman numerals I, II, and III correspond to the training schedule in Fig. 1.

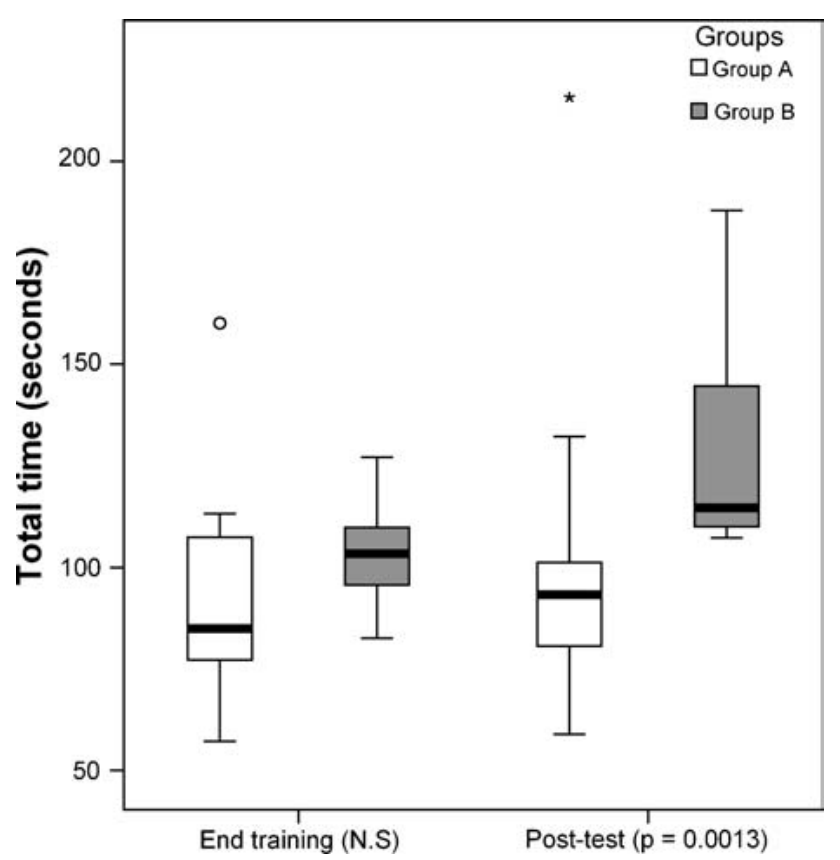

Fig. 3. Total time scores of groups A (training over several days) and B (training within a single day) for end training and after 7 days of no training on the posttraining test (posttest).

$(p=0.013)$. Group A tended to make fewer collisions and had a shorter path length for the right instrument and a longer path length for the left instrument, but these scores did not differ significantly from those for group B.

Comparison of the medians for each of the summed parameter scores between the training sessions showed no significant differences within the groups between repetition 4 to 5 and repetition 8 to 9 . Between these repetitions, both groups received a period of no training: 20 to $24 \mathrm{~h}$ for group A and 15 min for group B.
Analyses of the scores for each task separately showed that the time score significantly differed between the groups for drop the balls $(p=0.023)$ and the ring $(p=0.049)$, but not for the $30^{\circ}$ endoscope (Fig. 4a-c). Furthermore, other parameter scores (for collisions and path length) did not differ significantly.

\section{Discussion}

In the current study, the time scores show that the group training over several days was $18.7 \%$ faster than the group training within a single day when tested 7 days after the last training session. There were no statistically significant differences between the two groups in other performance scores such as collisions and path length.

Although it is a common adage that "practice makes perfect," recent studies suggest that training alone is not the only determinant of motor skills learning. The elapsed time between two repetitions also seems to have an important influence. Evidence from general motor skills research and endoscopic psychomotor skills has demonstrated that distributed training is superior to massed training $[4,12]$.

Skill acquisition is defined as the gain in performance during the training itself, during either distributed or massed schedules. The degree to which an acquired skill is retained over the passage of time is called skill retention [3]. The phenomenon of performance continuation in a stable state (permanent retention) or even improvement after training has ended is known as consolidation.

Other studies have shown that significant gains in motor performance are apparent in terms of both speed and accuracy when measured $24 \mathrm{~h}$ after training, even with no further training during the intervening interval $[2,15]$. The process of consolidation assumes long-term 
Table 2. Summed median scores for the posttraining test (posttest) for all tasks

\begin{tabular}{|c|c|c|c|c|c|}
\hline Parameter (medians) & & Group A & Group B & $\begin{array}{l}\text { Relative } \% \text { difference } \\
\text { between groups } \mathrm{A} \text { and } \mathrm{B}^{\mathrm{a}}\end{array}$ & $p$ Value \\
\hline \multirow[t]{3}{*}{ Time (s) } & First test & 305.9 & 247.5 & 23.6 & NS \\
\hline & End training & 85.0 & 103.0 & -17.5 & NS \\
\hline & Posttest & 93.3 & 114.8 & -18.7 & 0.013 \\
\hline \multirow[t]{3}{*}{ Collisions $(n)$} & First test & 16.0 & 13.0 & 23.1 & NS \\
\hline & End training & 3.0 & 4.0 & -25.5 & NS \\
\hline & Posttest & 4.0 & 4.5 & -11.1 & NS \\
\hline \multirow{3}{*}{ Path length: right (AU) } & First test & 64.2 & 77.9 & -17.6 & NS \\
\hline & End training & 51.8 & 48.6 & 6.5 & NS \\
\hline & Post test & 50.3 & 52.9 & -5.0 & NS \\
\hline \multirow{3}{*}{ Path length: left (AU) } & First test & 32.1 & 33.8 & -5.3 & NS \\
\hline & End training & 18.3 & 16.5 & 10.9 & NS \\
\hline & Post test & 20.7 & 19.2 & 7.8 & NS \\
\hline
\end{tabular}

AU, arbitrary units

First test: summated scores for the first training session

End training: summated scores for the 12th training session

Posttest: summated scores for test 7 days after the 12th training session

${ }^{a}$ (Score group $\mathrm{A}$ - score group $\mathrm{B}$ )/score group $\mathrm{B} \times 100 \%=$ relative difference

neurophysiologic changes that allow for the relatively permanent retention of learned behavior [7].

There is no clear explanation for why distributed training tends to be more effective and enhances consolidation, but it seems that during the rest periods, changes take place in the brain. It is known that motor skill improvement is sleep dependent [17, 18], and that rest results in better consolidation. Apparently, the brain needs rest periods to store learned motor skills adequately and to prevent fatigue or the possible effects of boredom. This may have important implications for the learning of psychomotor skills needed to perform endoscopic surgery. However, these findings apply for the learning of direct motor skills (e.g., tapping of fingers) and differ partly from findings that apply for the learning of psychomotor skills for endoscopic surgery.

In our study, there was no significant improvement in performance scores between the training blocks of four repetitions in the two training schedules. Neither short nor long breaks enhanced performance significantly during skill acquisition. Also, no difference in performance gain was found within the groups measured at the end of the training (end training). However, the posttraining test (posttest), 7 days after the last training, showed that performance time had increased slightly for both groups. Nevertheless, the increase in group B was significantly greater than in group A (i.e., group A was faster than group B). Thus, training with a break involving a night of sleep improves retention for performance time.

No significant difference was observed regarding the number of collisions and the path length score between the two groups. The exact reason for this remains unclear. The most likely reason concerning the collisions would seem to be a cognitive component in learning to avoid nontarget structures. In the simulator used, each time a collision occurred, the user was directly warned by a sound. The direct sound warning was used as a reminder to subjects that they should work carefully, and also as an alternative to force feedback within this simulator. As a consequence, the subjects quickly learned to reduce the collisions and seemed to pay less attention to the other parameters. Although both groups had the same number of collisions and the same path lengths, the distributed group (group A) performed the exercises faster. Therefore, training scheduled over several days is preferable for the training of endoscopic psychomotor skills.

An alternative explanation could be that the participants did not have enough training repetitions, and therefore were not completely out of their learning curve. Krakauer et al. [10], using a cursor dexterity task on a computer screen in which trainees did not see their hands, found that more initial training resulted in better retention. Hence, more repetitions of the tasks before the posttest could have increased the difference between groups A and B. The study of Krakauer et al. [10] also showed that a longer interval between the repetitions (skills acquisition) than $24 \mathrm{~h}$ is less effective.

With respect to path length, the decrease in score after 7 days was less than for time and collisions (Table 2). Trainees inexperienced in endoscopic techniques tended to make a lot of inadequate movements, especially at the beginning of the training. However, the skills acquisition in terms of path length occurred relatively fast and had good retention. Hence, the most effective way to bring the instrument from one place to the other was learned fast and stored more easily in the motor memory.

The study results support the use of a training schedule distributed over several days for the learning of endoscopic motor skills. However, for practical reasons, training lasting 2 days or more is not always possible. Most "nonuniversity" training hospitals lack adequate training equipment, or the structural organization of such training sessions frequently interferes with the busy clinical setting. Our results show that performance scores decreased up to $19 \%$ within a week. Taking into account that motor skills for endoscopic surgery may decrease to $81 \%$ within a month [19] if not used 

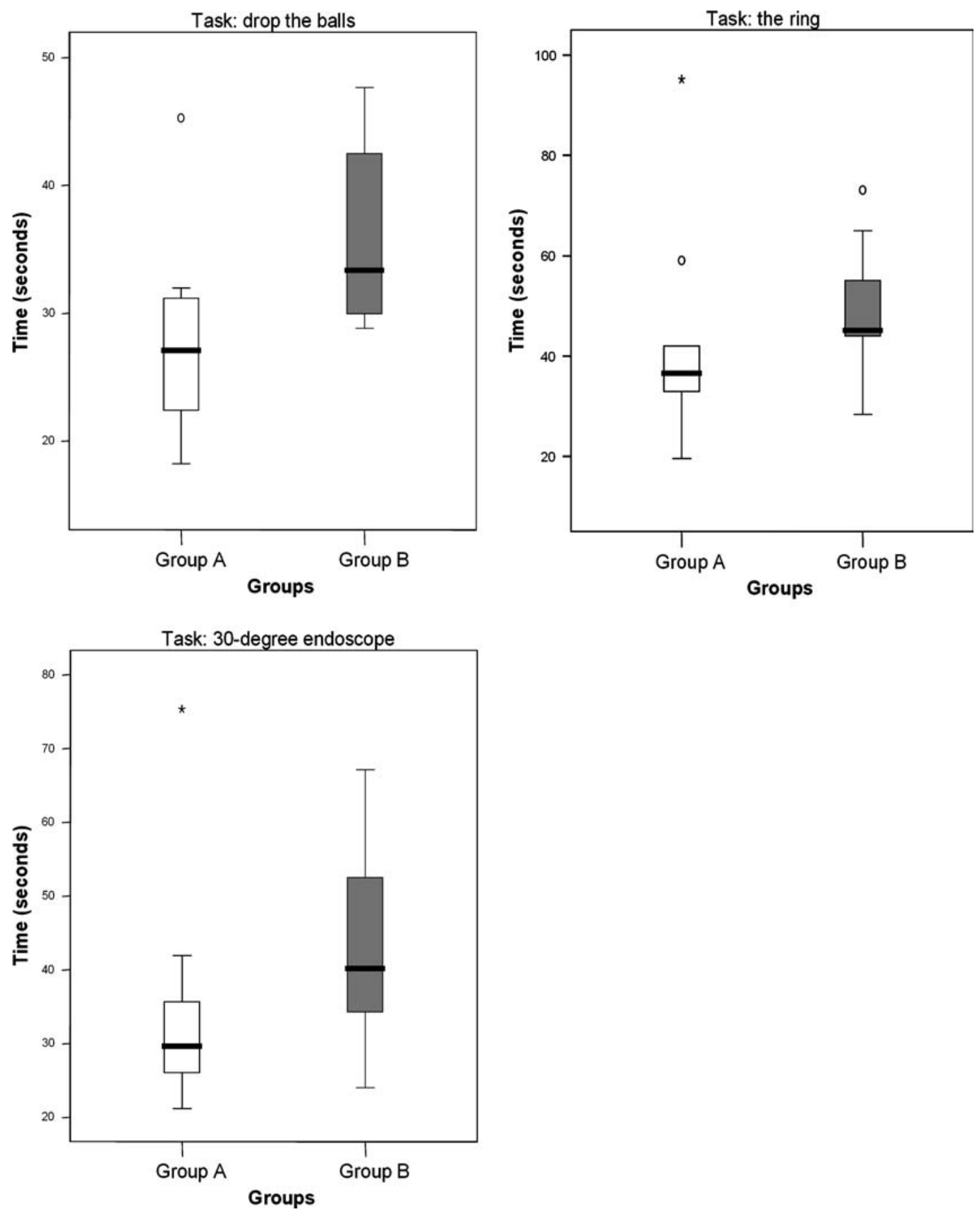

Fig. 4. Difference in time scores on the posttraining test between groups A (training on several days) and B (training on 1 day) after 7 days of no training for the following tasks: drop the balls $(\mathrm{A}<\mathrm{B} ; p=0.023)$, the ring $(\mathrm{A}<\mathrm{B} ; p=0.049), 30^{\circ}$ endoscope $(\mathrm{A}<\mathrm{B}$, nonsignificant difference).

frequently, trainees should be advised to perform their first procedure within at least 1 week after the course or receive training aimed at maintenance of their motor skills.

A paradigm shift from strictly criterion-based training (i.e., a trainee should show competence by attaining certain scores in the simulation) to the application of a training schedule that results in optimal skills retention over time seems warranted. The next step in research should be to investigate what effect distributed training schedules has is on long-term retention and consolidation of endoscopic motor skills.

\section{Conclusion}

The group with training distributed over several days was faster, with the same number of errors and instrument path length used. Psychomotor skill training for endoscopic surgery distributed over several days is 
superior to training within 1 day. Further research should focus on studying the long-term effects of distributed psychomotor skills training.

\section{References}

1. Bock O, Schneider S, Bloomberg J (2001) Conditions for interference versus facilitation during sequential sensorimotor adaptation. Brain Res 138: 359-365

2. Brashers-Krug T, Shadmehr R, Bizzi E (1996) Consolidation in human motor memory. Nature 382: 252-255

3. Dar-el E (2000) Human learning: from learning curves to learning organizations. Kluwer Academic Publishers, Haifa

4. Donovan JR, Radosevich DJ (1999) A meta-analytic review of the distribution of practice effect: now you see it, now you don't. J Appl Psychol 84: 795-805

5. Duffy AJ, Hogle NJ, McCarthy H, Lew JI, Egan A, Christos P, Fowler DL (2005) Construct validity for the LAPSIM laparoscopic surgical simulator. Surg Endosc 19: 401-405

6. Gallagher AG, Lederman AB, McGlade K, Satava RM, Smith CD (2004) Discriminative validity of the Minimally Invasive Surgical Trainer in Virtual Reality (MIST-VR) using criteria levels based on expert performance. Surg Endosc 18: 660-665

7. Gallagher AG, Ritter EM, Champion H, Higgins G, Fried MP, Moses G, Smith CD, Satava RM (2005) Virtual reality simulation for the operating room: proficiency-based training as a paradigm shift in surgical skills training. Ann Surg 241: 364-372

8. Grantcharov TPB, Funch-Jensen L, Rosenberg P (2003) Learning curves and impact of previous operative experience on performance on a virtual reality simulator to test laparoscopic surgical skills. Am J Surg 185: 146-149
9. Hyltander A, Liljegren E, Rhodin PH, Lonroth H (2002) The transfer of basic skills learned in a laparoscopic simulator to the operating room. Surg Endosc 16: 1324-1328

10. Krakauer JW, Ghez C, Ghilardi MF (2005) Adaptation to visuomotor transformations: consolidation, interference, and forgetting. J Neurosci 25: 473-478

11. Lee TD, Genovese ED (1988) Distribution of practice in motor skill acquisition: learning and performance effects reconsidered. Research Quarterly 59: 277-287

12. Mackay S, Morgan P, Datta V, Chang A, Darzi A (2002) Practice distribution in procedural skills training: a randomized controlled trial. Surg Endosc 16: 957-961

13. Schijven M, Jakimowicz J (2003) Construct validity: experts and novices performing on the Xitact LS500 laparoscopy simulator. Surg Endosc 17: 803-810

14. Seymour NE, Gallagher AG, Roman SA, O'Brien MK, Bansal VK, Andersen DK, Satava RM (2002) Virtual reality training improves operating room performance: results of a randomized, double-blinded study. Ann Surg 236: 458-463

15. Shadmehr R, Brashers-Krug T (1997) Functional stages in the formation of human long-term motor memory. J Neurosci 17: 409-419

16. Verdaasdonk EGG, Stassen LPS, Monteny L, Dankelman J (2005) Validation of a new and simple virtual reality simulator for training of basic endoscopic skills. The SIMENDO. Surg Endosc 20: $511-518$

17. Walker MB, Morgan A, Hobson JA, Stickgold R (2002) Practice with sleep makes perfect: sleep-dependent motor skill learning. Neuron 35: 205-211

18. Walker MP, Stickgold R, Alsop D, Gaab N, Schlaug G (2005) Sleep-dependent motor memory plasticity in the human brain. Neuroscience 133: 911-917

19. Windsor JA, Zoha F (2005) The laparoscopic performance of novice surgical trainees: testing for acquisition, loss, and reacquisition of psychomotor skills. Surg Endosc 19: 1058-1063 